\title{
A Case Study on Pemphigus Vulgaris
}

\author{
Bandaru Nagaraju*, Anne Ramu, Suryadevara vidhyadhara, Hanumanula Gunasree, Chadalavada \\ Arun Kumar
}

Department of Pharmacology, Chebrolu Hanumaiah Institute of Pharmaceutical Sciences, Chowdavaram, Guntur-19, A.P, INDIA.

\begin{abstract}
Pemphigus vulgaris is a rare chronic blistering skin disease. It is classified as a type II hypersensitivity reaction, with the formation of antibodies against desmosomes, components of the skin that function to keep certain layers of skin bound to each other. As desmosomes are attacked, the layer of skin separate and the clinical picture resembles a blister. Over time the condition inevitably progresses without treatment lesions increase in size and distribution throughout the body, behaving physiologically like a severe burn.
\end{abstract}

Key words: Vulgaris, Blistering, Hypersensitivity, Desmosomes, Lesions.

\section{INTRODUCTION}

The term "Pemphigus" is derived from the greek word pemphix (bubble or blister) ${ }^{1}$ and vulgaris derived from latin word (common). ${ }^{2}$ Pemphigus is a potentially life threatening disease that causes erosions and blisters of the skin and mucous membranes. ${ }^{3}$ Pemphigus Vulgaris(PV) is a chronic, rare, intraepidermal bullous disease with a potentially fatal outcome and originally named by Wickman in 1791. Pemphigus is an uncommon disease an incidence rate ranging from 0.5 to 3.2 per 100,000 per year. ${ }^{4}$ Men and women are equally affected .The most cases are occurring at the age of 50-60 years.

Pemphigus Vulgaris is a chronic mucocutaneous disease which usually manifests first in the oral cavity, which later may spread to skin or other mucous membrane. As it is a life threatening disease, it is important that dentist is able to recognize oral manifestations of PV and treat and refer appropriately. ${ }^{5}$ Pemphigus Vulgaris is an immunopathologic dermatologic disease that usually occurs in patients between the ages of 30 and 60. It is characterized by the development of flaccid, easily ruptured intraepithelial bullae on apparently normal skin and mucous membranes. The oral cavity is frequently affected in the course of the disease. Intraoral lesions may appear in as many as $50 \%$ of the patients without a simultaneous affection of the skin. Although any part of the oral mucosa may be affected, areas exposed to mechanical irritation are most commonly involved. The lesions tend to occur most frequently on the buccal and palatal mucosa and on the gingiva. The oral lesions begin as blebl ike blisters or as diffuse gelatinous plaques. Rupture of the bullae occurs in an early stage and may be caused by slight rubbing or minimal mucosal trauma. The lesions are usually painful. Untreated generalized Pemphigus Vulgaris may be fatal. Therefore, by recognizing the oral lesions of Pemphigus Vulgaris, the clinician has a responsibility in the early diagnosis of the disease, which is of the utmost prognostic importance. ${ }^{6}$

\section{CASE REPORT}

A $30 \mathrm{yr}$ female patient was admitted in dermatology department with chief complaints of fluid filled lesions over body since 2 days. Present complaint started 2 yrs back as fluid filled lesions on back later spread all over the body and later on the rapture and cause erosion. History of present illness is itching, burning sensation present over the erosions. Also had a history of fever, photosensitivity
DOI: 10.5530/ijopp.9.4.9

Address for correspondence: B. Nagaraju Assistant Professor, Department of Pharmacology, ChebroluHanumaiah Institute of Pharmaceutical Sciences, Chowdavaram, Guntur-19, A.P, India.

Phone no:9603674173

E-mail: bnrajupharma@gmail. com.

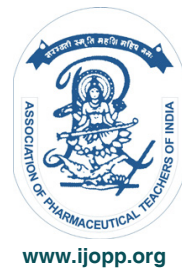


and joint pains. She has taken treatment outside hospitals. No similar complaints in the past. No history of DM and HTN and surgery done for Sinusitis. Systemic examination

\section{CVS}

Normal S1 and S2 heart sounds. Musculoskeletal systemArthralgia's present. Dermatological examination: Hyper pigmented plaques with crusting are present over anterior and posterior aspect of trunk tiny erosions positive. Few hyper pigmented plaques with erosions are seen over both thighs. Face: few tiny crusted small plaques are present over chin, submandibular region. Scalp: hyper pigmented crusted small plaques seen over scalp. Nails: acute paronychia present over at fingers. Oral mucosa: Tiny erosions over hard palate buccal mucosa present.

\section{Diagnosis}

Temperature: $98.4 \mathrm{f}$ Pulse rate: 90, Respiratory rate: 22, BP: 120/80 mmHg Hb: 12.9 gm\%, WBC: 9,800cells/ cumm, RBC: 4.9 million cells/cumm, Platelets: 2.0 laks, ESR:10 mm/hr, Serum urea: $15 \mathrm{mg} / \mathrm{dl}$, Serum creatinine: $0.8 \mathrm{mg} / \mathrm{dl}$, sodium: $135 \mathrm{mmol} / \mathrm{l}$, Potassium: $3.8 \mathrm{mmol} / \mathrm{l}$, HbAIC: $5.4 \%$ and pus cells: 6-8 cells/h.p.f.

Based on objective and subjective parameters the patient is suffered with Pemphigus Vulgaris.

In this case the patient is treated with following mediations.

Medications; Inj:Dalacin-C(Clindamycin phosphate) antibacterial antibiotic $600 \mathrm{mg}$ BD, Inj:Lenzolid (Linezolid)antibiotic $500 \mathrm{mg} \mathrm{BD}$, Candid mouth paint L/A(Clotrimazole) antibacterial TID,Inj. Fluconazole (Fluconazole) antifungal $200 \mathrm{mg}$ OD,Inj. Optineuron (B-Complex) vitamin suppliment 1 vial OD,Cap. Fesovit (Ferrous sulphate, Folic acid and VitB) iron suppliment OD, Inj. Pantop (Pantoprazole) antiulcer 1 vial OD, Digene syrup (Aluminium Hydroxide) antiulcer 3 tsp TID,Tab. Astervit (Ascorbic acid, Vit C)vitamin supplement BD,Tab. Resodium (Tolvaptan) vasopressin receptor 2 antagonist $30 \mathrm{mg}$ OD, Tab. Taxim$\mathrm{O}$ (Cefixime) cephalosporin antibiotic $200 \mathrm{mg}$ BD, Tab.Dolo (Acetaminophen)anti pyretic $650 \mathrm{mg}$ SOS, Soframycin cream (Framycetin-Sulphate) antibacterial BD,Tab. Augmentin (Amoxicillin+Clavulanate)broad spectrum antibiotic $625 \mathrm{mg}$ TID, Tab. Zental (Albendazole) anti-amoebic 400 mgOD Tab. Wysolone (Prednisolone) corticosteroid $30 \mathrm{mg}$ OD, Emoderm cream L/A(White soft paraffin and liquid paraffin) emollient TID, Tab. Defza (Deflazacort)corticosteroid $36 \mathrm{mg} /$ day, Tab.Shelcal (calcium carbonate) calcium supplement
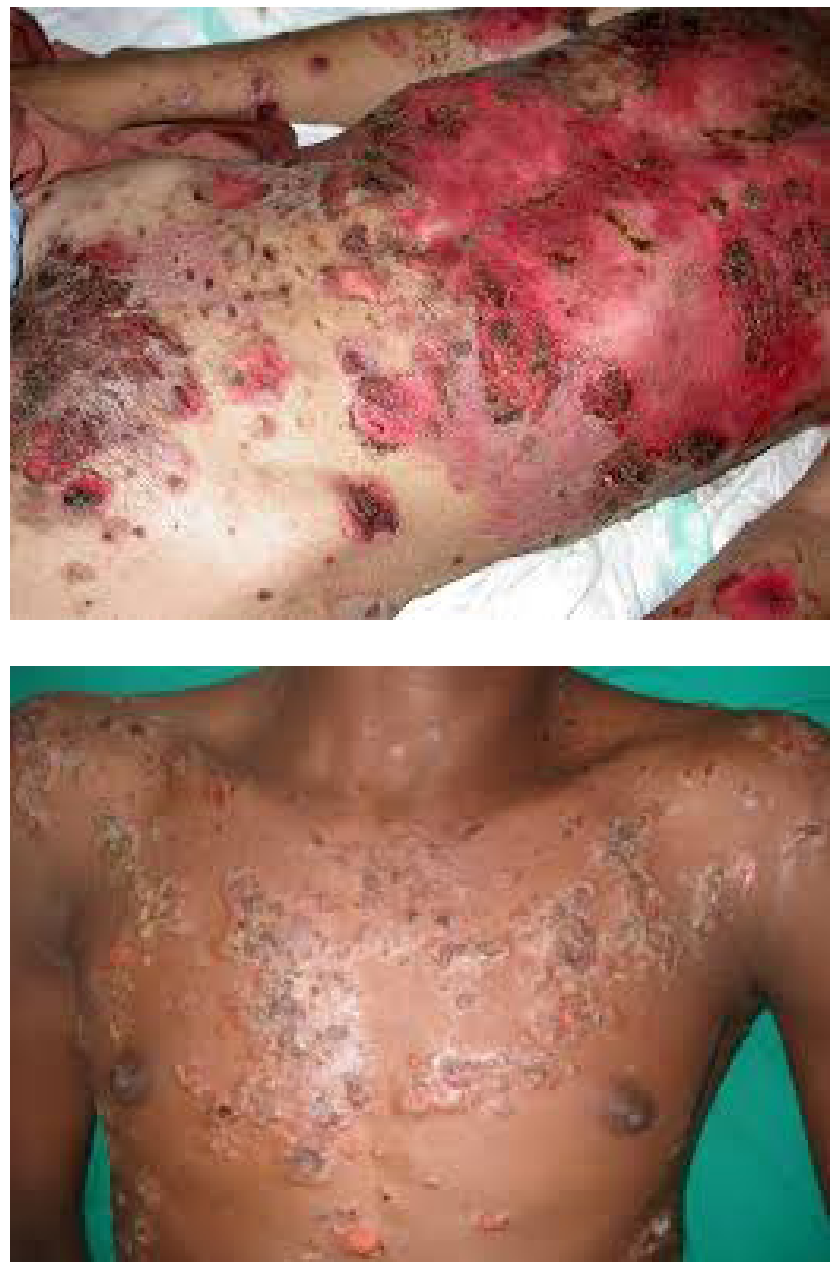

500 mg BD, Syp. Potklor Potassium chloride)potassium supplement $5 \mathrm{ml}$ TID.

\section{DISCUSSION}

In pemphigus vulgaris, lesions at first comprise small asymptomatic blisters, although these are very thinwalled, they easily rupture giving rise to painful and hemorrhagic erosions.70-90\% cases the first signs of disease appear on the oral mucosa. While lesions can be located anywhere within the oral cavity, they are most commonly found in areas are check, mucosa, tongue, palate and lower lip. The ulcerations may affect other membranes including the conjunctiva, nasal mucosa, pharynx, larynx, esophagus and genital mucosa, as well as the skin where blisters are commonly seen. ${ }^{7}$ Increased salivation and problems with chewing and swallowing are the major subjective complaints. ${ }^{8}$

The etiology of this case is still unknown. These groups of diseases are characterized by the production of antibodies against intercellular substances so, therefore, classified as autoimmune diseases. ${ }^{7}$ Other initiating factors reported included certain food (garlic),infections, neo- 
plasms and some drugs like captopril, penicillmine and rifampicin. ${ }^{9}$

In pemphigus vulgaris, auto antibodies are produced against desmosomes (adhesion proteins), specially desmoglein 3 (Dsg 3). Another important component of desmosomes is desmoglein 1 . The first target is effects on subcutaneous site only. Dsg 3 is expressed in oral mucosa and Dsg 1 expressed in skin. ${ }^{10}$

The principal dermal and mucosal changes involve the loss of coherence among layers of keratinocytes. This is manifested, in the early stages of diseases; the primary lesion is ath in walled bulla, several centimeters in size, containing clear fluid. Under pressure it releases its contents through the surrounding epidermis and further increases in size. Healing is very slow, but no scars will remain. On the oral mucosa, bullae filled with fluid are also present but no inflammation develops. When the epithelial wall of the bulla ruptures it will become painfull lesions. ${ }^{11}$

In this case, we assessed the Multiple drugs for single condition and initiation of new drug therapy to treat pemphigus vulgaris with more antibiotics were prescribed. So, inj. Linezolid and tab. Augmentin were avoided. Along with steroids to treat pemphigus, immuno suppressive agents were also prescribed such as azathioprine $100 \mathrm{mg}$ OD because the disease is of autoimmune origin. As Inj. Pantop is sufficient and Syp. Digene can be avoided.

\section{CONCLUSION}

Pemphigus vulgaris is a rare cause of chronic ulceration of mucosa. The severity and natural history of pemphigus vulgaris are variable, but before the advent of steroids, most patients with pemphigus vulgaris died. Treatment with systemic steroids has reduced the mortality rate dramatically. Untreated, pemphigus vulgaris is often fatal because of the susceptibility to infection and fluid and electrolyte disturbances. Most deaths occur during the first few years of disease, and, if the patient survives 5 years, the prognosis is good. Early disease probably is easier to control than widespread disease, and mortality rates may be higher if therapy is delayed.

\section{ACKNOWLEDGMENT}

The authors are thankful to NRI Hospital for the collecting of this case.

\section{CONFLICT OF INTEREST}

The author declares there is no conflict of interest.

\section{ABBREVIATION USED}

BP: Blood Pressure; PR: Pulse rate; RR: Respiratory rate; CV: Cardiovascular; WBC: White blood cells; RBC: Red blood cells; ESR: Erythrocyte Sedimentation rate.

\section{REFERENCES}

1. Shafer, Hine, Levy. Textbook of Oral Pathology $5^{\text {th }}$ edition Neville,Damn,Allen,Bouquet. Textbook of Oral and Maxillofacial pathology $2^{\text {nd }}$ edition. Philadeplphia 1995, saunders martin D Greenburg.Micheal Glick Burketts Orla medicine Diagnosis and treatment $10^{\text {th }}$ edition.

2. Korman N. Pemphigus J. Am Acad dermat. 1998;18;1219-38.

3. Ahmed AR. Lymphocyte studies in Pemphigus. Arch Dermatol Res. 1998;271:111-5. http://dx.doi.org/10.1007/BF00417395.

4. Pisanti S, Sharav Y, Kaufman E, Posner LN. Pemphigus vulgaris; incidence in jews of different ethnic groups according to age sex and initial lesions. Oral path oral med. Oral Sug. 1974;38(3);382-7. http://dx.doi.org/10.1016/00304220(74)90365-X .

5. Sreeshyla HS, Usha Hedge, Vidya GD. Oral pemphigus vulgaris-report of a case with review on it"s etiopathogenesis. Archives of oral sciences and research

6. Golchai J, Shams G. A familial case of pemphigus vulgaris. Medical journal of islamic republic of Iran. 1993;6(4)297-8.

7. Dagistan S, Georgen M, Miloglu O, Cakur B. Oral Pemphigus Vulgaris: A case report with review literature. J Oral Sci. 2008;50(3):359-62. http://dx.doi. org/10.2334/josnusd.50.359; PMid:18818476.

8. Huntley $\mathrm{AC}$, et al. pemphigus vulgaris and Vegetating and Verrucous lesions: Case Report. Dermatol Online J. 2004;9.

9. Fassmann A, Dvo.akova N, Izakoviava Holla L, vanuk J, Wotke J Manifestations of pemphigus vulgaris in the orofacial region.Case report script Medica2003;76:55-62.

10. Robinson NA, Yeo JF, Lee YS, Aw DC. Oral pemphigus vulgaris: A case report and review literature. Ann Acad Med Singapore. 2004:33;63-8. PMid:15389311.

11. Pradeep AR, Manojkumar ST, Arjun R. pemphigus vulgaris associated with significant periodontal findings: A case report. J Calif Dent Association. 2010;38(5):343-60. PMid:20572529. 